Original Paper http://ajol.info/index.php/ijbcs http://indexmedicus.afro.who.int

\title{
Effet des touffes de Hyphaene thebaica (Mart) sur la production du mil dans la région de Maradi (Niger)
}

\author{
Nomaou DAN LAMSO ${ }^{1 *}$, Yadji GUERO ${ }^{1}$, Abdourahamane TANKARI DAN-BADJO ${ }^{1}$, \\ Rabah LAMAR ${ }^{2}$, Babou ANDRE BATIONO ${ }^{3}$, Patrice DJAMEN ${ }^{4}$, Adamou \\ Didier TIDJANI ${ }^{1}$, Nassirou ADO MAMAN ${ }^{1}$ et Ambouta Jean Marie KARIMOU ${ }^{1}$ \\ ${ }^{I}$ Département Sciences du sol, Faculté d'Agronomie de Niamey, Université Abdou Moumouni de Niamey, \\ Niamey, Niger. \\ ${ }^{2}$ Centre de Coopération Internationale en Recherche Agronomique pour le Développement (CIRAD), \\ Montpellier, France. \\ ${ }^{3}$ Institut de l'Environnement et Recherches Agricoles (INERA), Ouagadougou, Burkina Faso. \\ ${ }^{4}$ African Conservation Tillage Initiative (ACT), Ouagadougou, Burkina Faso. \\ *Auteur correspondant, E-mail: danlamso@gmail.com; Tél.: (00227) 96596818
}

\section{RESUME}

La région de Maradi est confrontée à la saturation de l'espace et à la baisse généralisée de la fertilité des sols. Pour faire face à cette situation, les paysans ont adopté l'association céréales - légumineuses, l'apport des fertilisants par micro-doses et l'utilisation des ligneux dans les champs. Ainsi, dans le terroir de El Guéza, les céréales sont cultivées en association avec l'arbuste Hyphaene thebaica, très répandu dans la localité. C'est dans cette optique que cette étude a été conduite pour évaluer l'influence des touffes de Hyphaene thebaica sur la production du mil dans ce terroir. Ainsi, des essais de culture du mil ont été menés selon un dispositif en blocs randomisés complet sur 3 parcelles avec touffes de Hyphaene thebaica et une parcelle témoin (sans touffes). Les résultats obtenus ont révélé qu'autour de la touffe de Hyphaene thebaica, le mil présente un enracinement très dense et bien développé alors qu'hors Hyphaene thebaica, il a des racines fines, moins denses et développées latéralement. Par ailleurs, les rendements en grains et en paille autour de Hyphaene thebaica sont respectivement de 1300 à $1820 \mathrm{~kg} / \mathrm{ha}$ et 4630 à $6740 \mathrm{~kg} / \mathrm{ha}$ alors qu'ils sont de $440 \mathrm{~kg} / \mathrm{ha}$ et 2000 $\mathrm{kg} / \mathrm{ha}$ pour le témoin. Cette étude montre clairement que les touffes de Hyphaene thebaica améliorent la production du mil.

() 2015 International Formulae Group. All rights reserved.

Mots clés : Touffes, Hyphaene thebaica, mil, rendements, El Guéza, Maradi.

\section{Effect of Hyphaene thebaica (Mart) tufts on the millet production in Maradi region (Niger)}

\begin{abstract}
The region Maradi is facing the land saturation and the general decline of soil fertility. To cope, farmers have adopted several techniques like "association cereals - legumes", input of micro-doses fertilizers and the use of woody species in the fields. Thus, in the land of El Guéza, cereal crops grains are grown in combination
\end{abstract}


with Hyphaene thebaica shrub, widespread in this locality. It is in this light that this study was conducted to evaluate the influence of Hyphaene thebaica tufts on millet production in this region. Thus, millet cultivation trials were conducted according to a randomized complete block design of 3 plots with Hyphaene thebaica tufts and a control plot (without tufts). The results obtained revealed that around the tufts of Hyphaene thebaica, millet has a very dense root and well developed whereas outside Hyphaene thebaica, millet has fine roots, less denses and developed laterally. Moreover, the yields of grain and straw around Hyphaene thebaica are $1300-1820 \mathrm{~kg}$ / ha from 4630 to $6740 \mathrm{~kg}$ / ha while they are $440 \mathrm{~kg}$ / ha and 2,000 kg / ha for the control respectively. This study clearly shows that the tufts of Hyphaene thebaica improve millet production.

(c) 2015 International Formulae Group. All rights reserved.

Keywords: Tufts, Hyphaene thebaica, millet, yields, El Guéza, Maradi.

\section{INTRODUCTION}

Dans les zones arides et semi-arides, les problèmes de dégradation des terres se posent avec acuité et engendrent une baisse de la fertilité des terres agricoles (Roose, 1974 ; Dan Lamso, 2002). Pour restaurer ces terres et améliorer leur fertilité, outre les techniques classiques de conservation des eaux et des sols telles que les cordons pierreux, le zaï, et les demi -lunes, par exemple, des espèces végétales notamment les espèces ligneuses sont aussi utilisées (Bationo et al., 2012).

$\mathrm{Au}$ Niger, l'agriculture en régime pluvial est marquée par une forte utilisation de la matière organique comme fertilisant (Bationo et al., 2012; Ambouta et al., 1998 ; Evequoz et Guéro, 2000). Il s'agit du fumier, des ordures ménagères et autres déchets pour les champs de case proches des habitations constituant la première auréole de fertilité et sans arbustes (Dramé et Berti, 2008). Par contre, dans les champs éloignés de ces habitations, communément appelés champs de brousse (Marchal, 1983), les apports précédents sont négligeables mais les rejets des souches coupés lors de la préparation des champs en fin de saison sèche (mars - avril) et au cours de la campagne agricole, constituent les principales sources de matière organique (De Grandi, 1996). Ces rejets luttent contre l'érosion éolienne pendant la saison sèche alors que le sol est nu (Peltier et al., 2008; Bationo et al., 2012). Des travaux de recherche ont porté sur l'influence de Faidherbia albida (Maï Moussa, 1996; Мaï Moussa et al., 1997), Hyphaene thebaica (Moussa, 1997), et Borassus aethiopum (Kadadé, 1999) en tant qu'arbres, sur la production du mil, mais peu se sont intéressés sur le rôle des arbustes dans l'amélioration de la production du mil (Moussa, 1997). Des recherches récentes (Dan Lamso et al., 2015) ont montré une amélioration de la fertilité des sols en présence des touffes de Hyphaene thebaica. En vue donc de mettre en évidence l'effet de cette amélioration sur la production du mil, une étude est menée dans le terroir de El Guéza. Elle a pour objectifs de suivre la phénologie et de déterminer le rendement du mil autour des touffes de Hyphaene thebaica, qui est une espèce très répandue dans la région de Maradi notamment dans le Goulbi N'Kaba, où elle s'est imposée comme espèce agroforestière très appréciée par les paysans.

\section{MATERIEL ET METHODES}

\section{Présentation et choix du site d'étude}

L'étude s'est déroulée dans le terroir villageois de El Guéza situé à $12 \mathrm{~km}$ au Sud de Gazaoua, chef lieu de département (cf Figure 1). Il est limité à l'Ouest par le terroir de Rogogo, à l'Est par celui de Dan Damou et Bougouzawa, au Sud-Est par le terroir de Guidan Adamou, au Sud par Touloubouché et au Nord par le terroir de Oungouwa Landi. 
A l'instar de la région de Maradi, le climat de ce terroir est de type sahélien avec une pluviométrique annuelle moyenne comprise entre 400 et $650 \mathrm{~mm}$. L'agriculture pluviale constitue ainsi la principale activité socio-économique des populations. Les cultures dominantes sont le mil, le sorgho, le niébé et l'arachide et secondairement le sésame, le voandzou et l'oseille. Cependant, cette activité connaît plusieurs contraintes notamment la baisse de la fertilité des sols, une crise foncière agricole, l'insuffisance et l'irrégularité des pluies et des pressions parasitaires. On note aussi une très faible mécanisation de l'agriculture et un faible accès aux autres intrants agricoles.

Les sols du terroir sont dominés par les sols ferrugineux tropicaux développés d'une part sur du sable et d'autre part sur des placages argilo-sableux. Ces sols sont appelés respectivement «Jigawa » et « Guéza ou Fadama» (Ambouta et al., 1998). Le couvert végétal du terroir est composé de Faidherbia Albida, Prosopis africana, Hyphaene thebaica, Piliostigma reticulatum.

Le choix du terroir de El Guéza a été fait selon les critères suivants: densité de Hyphaene thebaica, parcelles expérimentales non loin du village, et devant représenter le système de culture dominant du terroir. A cet effet, un champ présentant une densité de 204 touffes/ ha de Hyphaene thebaica et cultivé selon une association mil-sorgho-niébé, a été choisi.

\section{Matériel végétal}

\section{Le matériel végétal est composé de}

Hyphaene thebaica qui est une espèce de la famille des Arecacea, de la tribu des Borasseae, du genre Hyphaene, espèce thebaica, caracterisée par un stipe annelé en forme d'Y rappelant son architecture apparentée au modèle de schoute, c'est-à-dire les arbres à ramification dichotomique (Jahiel,
1993). Selon Maydell (1981), Hyphaene thebaica est une espèce à croissance dichotomique très lente : une première fructification n'a lieu qu'après 6 à 8 ans et l'âge de la révolution est de 60 à 80 ans, avec apparition d'un renflement à partir de la $25^{\text {ème }}$ année. Le peuplement de Hyphaene thebaica se rencontre dans les steppes et les fourrés sahéliens et soudano-sahélien sur les sols sableux à $\mathrm{pH}$ basique ou neutre, dans les bas fonds inondables et autour des villages dans les dépressions sableuses (Cissé et al., 2005). $\mathrm{Au}$ Niger, Hyphaene thebaica se rencontre dans les dallols, les goulbis et dans la vallée $\mathrm{du}$ fleuve Niger où il forme des parcs agroforestiers (Ounteini, 1993). C'est une espèce thermophile qui supporte des températures moyennes annuelles élevées $\left(>30{ }^{\circ} \mathrm{C}\right)$ et des déficits de saturation très élevés. Dans les zones à faibles précipitations (<30 mm), sa localisation se limite aux sites possédant une nappe phréatique subaffleurante (Harouna, 2005). La caractérisation du peuplement de Hyphaene thebaica dans le terroir de El Guéza a été faite à partir des observations de terrain (Figure 2).

Pennisetum glaucum (ou petit mil) : il s'agit de la variété de mil CT6 mise au point par l'Institut National de Recherches Agronomiques (INRAN) du Niger en 1995 avec un cycle de 55 à 65 jours et un rendement potentiel de $2000 \mathrm{Kg} / \mathrm{ha}$.

\section{Dispositif expérimental}

Il s'agit d'un dispositif en blocs randomisés complet avec trois répétitions des touffes de Hyphaene thebaica. Le choix de la touffe (ou bélou) s'est porté sur son isolement par rapport à une autre touffe et/ou un autre ligneux. Ainsi, les trois (3) touffes choisies sont distantes l'une de l'autre d'au moins 50 m.

Les quatre côtés (Est, Ouest, Sud et Nord) de chaque touffe sont considérés 
comme traitements du dispositif. Ainsi, les parcelles TE, TO, TN et TS d'une superficie de $9 \mathrm{~m}^{2}$ chacune, et respectivement pour les côtés Est, Ouest, Nord et Sud ont été placées au niveau de chaque répétition. Etant donné que les touffes des arbustes contribuent à lutter contre l'érosion éolienne, le choix des 4 points cardinaux permet de mesurer l'effet (sur la production de mil autour des touffes) des deux vents dominants, la mousson et l'harmattan, qui s'opposent par leurs directions. Une parcelle Témoin sans touffes de Hyphaene thebaica a été aussi choisie. La Figure 3 donne le schéma du dispositif expérimental.

\section{Observations et mesures sur la culture du mil}

Elles se rapportent :

- aux mesures hebdomadaires de la hauteur des plants de la montaison à la maturité physiologique à l'aide d'une règle;

- au constat des dates de $50 \%$ de levée, de tallage, de montaison, d'épiaison, de floraison et de maturité physiologique sur chaque sous parcelle ;

- à l'évaluation des composantes du rendement (poids des grains et de la paille sèche) au niveau de chaque touffe de Hyphaene thebaica. En effet, la récolte a été effectuée à travers un recepage total des pieds de mil et après quatre (4) jours de séchage, les épis ont été coupés puis décortiqués. Ainsi, les rendements en grains et en tiges ont été évalués ;

- à la notation des attaques parasitaires.
Ces observations et mesures ont été faites autour des touffes suivant les 4 directions géographiques (Est - Ouest et Nord - Sud).

Etude du système racinaire des ligneux et de la culture du mil

L'étude de l'architecture des racines de Hyphaene thebaïca, en présence de la culture du mil est menée selon la méthode de déblayage latéral (Figure 4). Le travail consistait à déblayer (sans couper les racines ni leur changer de disposition) une touffe de Hyphaene thebaica associée au mil. L'opération est répétée mais sur des pieds séparés. L'objectif est d'apprécier la distribution des racines, leur développement et leur organisation.

La Figure 4 montre que la touffe de Hyphaene thebaica est quasiment déterrée pour faire ressortir le maximum des racines.

\section{Analyse statistique des données}

Les données du suivi phénologique ont été traitées à l'aide du logiciel GenSTAT. Ainsi, les données obtenues ont été soumises à une analyse de variance pour identifier la présence ou non de différences significatives (au seuil de $5 \%$ de probabilité) pour les différents paramètres selon les 4 points cardinaux. En présence de différences significatives, le test de Student Newman Keuls (au seuil de 5\%) a été effectué pour identifier les traitements significativement différents selon la variable considérée. 


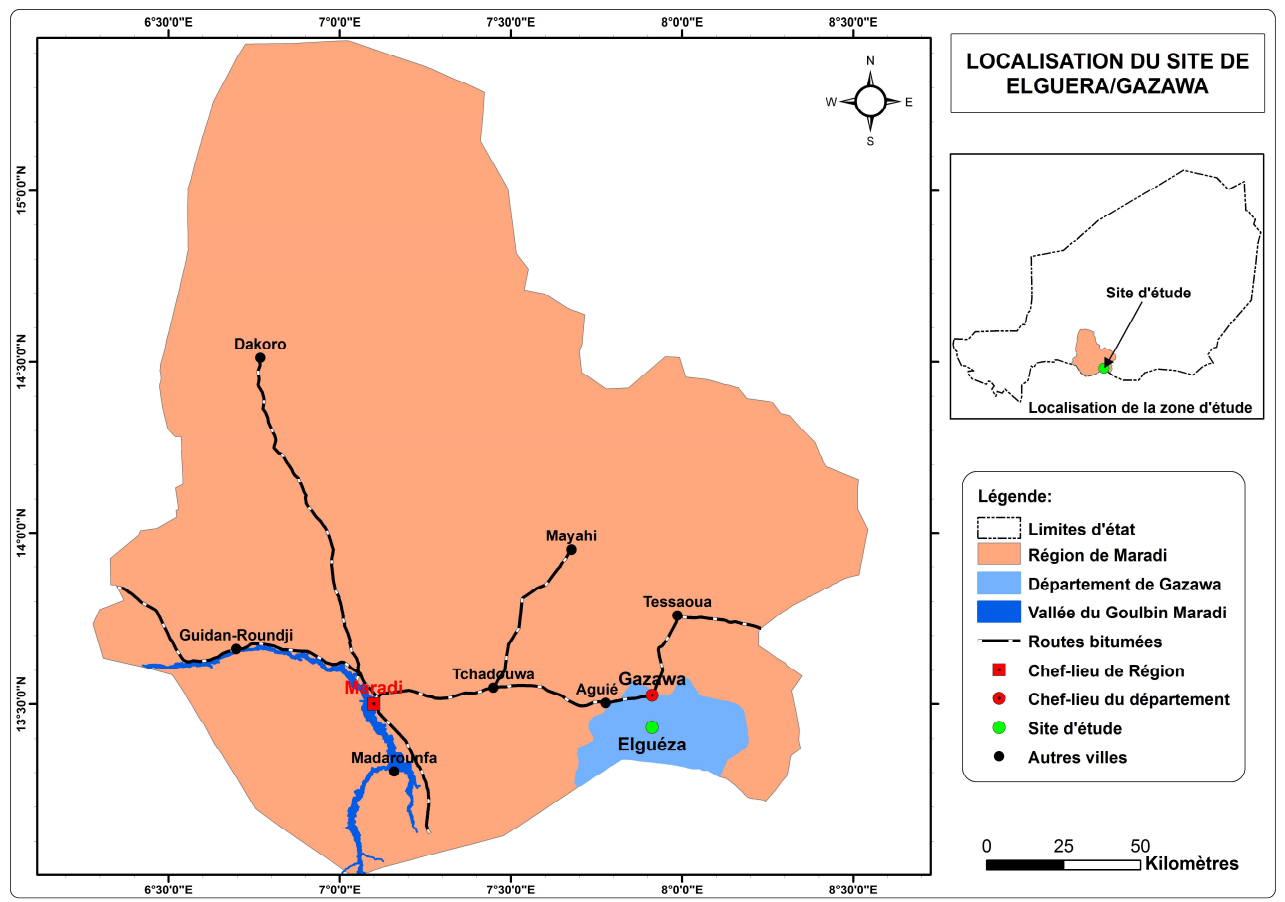

Figure 1 : Localisation du site de El Guéza (Issaka Moussa, DSS/FA/UAM).

\section{RESULTATS ET DISCUSSION}

Développement des systèmes racinaires de Hyphaene thebaïca et du mil

La Figure 5 illustre l'architecture racinaire du mil en présence et en absence de Hyphaene thebaica.

Après le déblayage, on distingue trois (3) types de racines selon leur disposition :

- des racines qui prospectent le sol verticalement à la recherche de la nappe phréatique. Elles sont nombreuses et de taille variable. On les rencontre lors de fonçage des puits ;

- des racines latérales de direction oblique qui s'enfoncent dans le sol jusqu'à 1 $\mathrm{m}$. Elles sont généralement peu nombreuses, plus ou moins grosses et peuvent avoir jusqu'à $16 \mathrm{~m}$ de long ;

- des racines superficielles se développant latéralement et qui émettent généralement des bourgeons pouvant donner naissance à d'autres plants. Elles sont le plus souvent de taille moyenne et sont relativement nombreuses.
D'après les constats des paysans, les racines de Hyphaene thebaica ont une grande capacité de descendre plus profondément à la recherche de l'eau. Hyphaene thebaica serait un bon indicateur des milieux humides.

Les observations de terrain ont permis aussi de constater que le mil cultivé autour de la touffe de Hyphaene thebaica présente un enracinement très dense et bien développé. Les racines ont tendance à explorer des horizons plus profonds $(40-60 \mathrm{~cm})$.

En ce qui concerne le mil hors Hyphaene thebaica, les racines sont fines, superficielles (20 à $40 \mathrm{~cm}$ ), moins denses et se développent latéralement contrairement au cas précédent. Le bon développement du mil associé à Hyphaene thebaïca serait dû à la restitution d'une partie de l'eau à cette culture par Hyphaene thebaica à laquelle il faut ajouter une meilleure disponibilité en azote et en phosphore comme l'ont démontré des travaux antérieurs (Dan Lamso et al., 2015). 
Il apparaît clairement que les touffes de Hyphaene thebaica ont une influence positive sur le développement racinaire du mil.

Influence des touffes de Hyphaene thebaica sur les paramètres de production du mil Production de talles

Le Tableau 1 affiche la production totale moyenne de talles selon les directions, comparée au témoin ainsi que la proportion des talles fertiles.

La production totale moyenne des talles autour des touffes de Hyphaene thebaïca est variable. En effet, elle est 2 à 3 fois plus élevée que celle du témoin. Quant à la proportion des talles fertiles, elle est plus importante selon les directions Est - Ouest contrairement aux talles totales.

\section{Croissance du mil}

La croissance du mil est illustrée par la Figure 6. La hauteur des tiges du mil à 33, 40, 47 et 54 jours après semis (JAS) a été relevée sur toutes les parcelles. La culture du mil a une croissance plus ou moins accélérée selon les parcelles.

Il ressort de la Figure 6 que la hauteur des tiges du mil dans les parcelles couvertes de Hyphaene thebaica est nettement supérieure à celle enregistrée dans la parcelle témoin. En effet, à 54 JAS (25 août), la hauteur des tiges relevée dans les parcelles couvertes de Hyphaene thebaica $(72$ à $98 \mathrm{~cm})$ est de 22 à $42 \%$ plus élevée que celle enregistrée dans la parcelle témoin $(56 \mathrm{~cm})$. Aussi, on observe toujours la prédominance des directions Est - Ouest. L'importance des teneurs en éléments chimiques notamment le $\mathrm{N}$ total, le $\mathrm{P}$ assimilable et surtout le $\mathrm{Ca}$ et le $\mathrm{Mg}$ échangeables au niveau de Hyphaene thebaica serait probablement à l'origine de cette croissance. En effet, le calcium et le magnésium jouent un rôle physiologique important pour les plantes et représentent normalement à eux seuls 70 à $95 \%$ des éléments minéraux les plus disponibles pour la plante (Boyer, 1978). De même, la touffe de Hyphaene thebaica génère à travers sa partie aérienne et son système racinaire un microclimat qui favoriserait la croissance du mil. Les hauteurs des tiges enregistrées au niveau des parcelles couvertes de Hyphaene thebaica ont fait l'objet d'une analyse de variance selon les facteurs temps et la position par rapport à la touffe. Cette analyse montre qu'il existe des différences significatives entre les parcelles $(\mathrm{F}=0,024)$ sur la croissance du mil dans le temps et selon la direction des vents dominants car la hauteur des tiges dans les parcelles TE et TO est beaucoup plus importante que celles des TN et TS.

Rendements en grains et en tiges de mil

La Figure 7 illustre les rendements en grains et en tiges de mil pour les parcelles autour de Hyphaene thebaica et le témoin.

L'analyse de la Figure 7 montre que les rendements en grains et en tiges de mil autour des touffes de Hyphaene thebaica sont nettement supérieurs à ceux du témoin. Pour les grains, ils sont 3 à 4 fois (1300 à 1820 $\mathrm{kg} / \mathrm{ha}$ ) plus importants que celui du témoin (440 kg/ha). Ces résultats sont proches de ceux de Moussa (1997) qui a obtenu une augmentation de 2 à 3 fois de rendements en grains de mil dans les parcelles sous influence de Hyphaene thebaica (800 à $1000 \mathrm{~kg} / \mathrm{ha}$ ) par rapport aux parcelles sans Hyphaene thebaica (350 kg/ha). Par contre, ils sont inférieurs à ceux de Kadadé (1999) qui a travaillé sur l'arbre Borassus aethiopum (rônier) et a obtenu de rendement en grains de mil de l'ordre de 700 à $2600 \mathrm{~kg} / \mathrm{ha}$. Des résultats similaires ont été aussi obtenus par Wezel (2000) qui a montré que l'environnement immédiat des arbustes en général, favorise une bonne production du mil.

Concernant les tiges, la production de la parcelle témoin est de $2000 \mathrm{~kg} / \mathrm{ha}$ représentant la moitié voire le tiers de celles des parcelles sous influence de Hyphaene thebaica (4630 à 6740 kg/ha).

L'analyse de variance effectuée a permis de relever des différences significatives entre les parcelles Nord - Sud et Est - Ouest qui sont sous l'influence des vents dominants. En effet, les rendements en grains et en tiges dans les parcelles Est et Ouest de la 
touffe sont significativement supérieurs à ceux des parcelles Nord - Sud. Cela serait donc lié aux apports de biomasse et de particules transportées et piégées par la touffe favorisant ainsi le développement de la culture de mil. Cette amélioration très significative de la production du mil par les touffes de Hyphaene thebaica est comparable à celle engendrée par certaines techniques physiques de conservation des eaux et des sols notamment le zaï, les cordons pierreux et les demi-lunes et pourrait, de ce fait, faire de ces touffes une solution de remplacement (dans un contexte généralisé de dégradation des terres) des techniques physiques dans les zones où elles sont difficiles à réaliser.

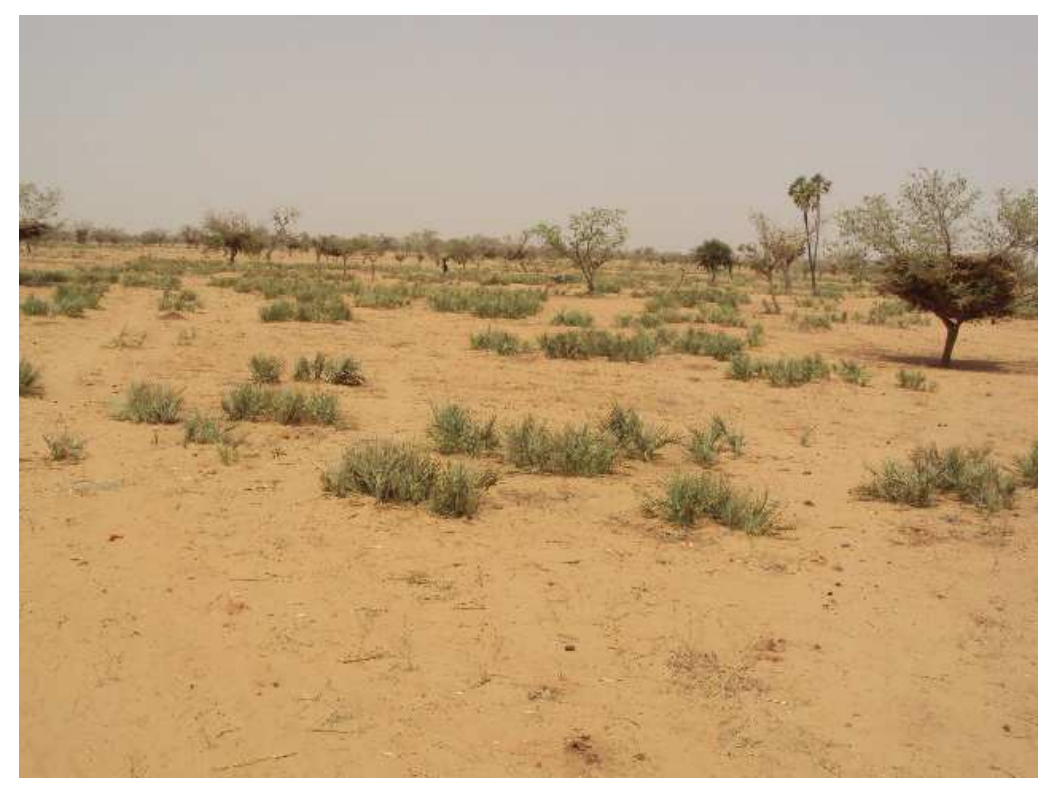

Figure 2 : Une vue des touffes de Hyphaene thebaica dans le terroir de El Guéza.

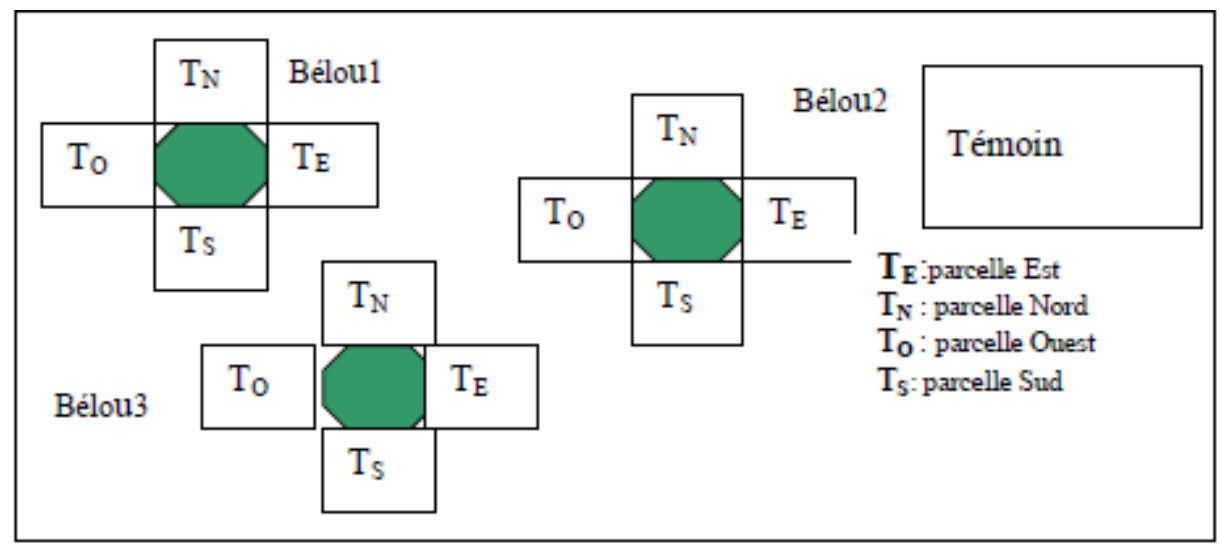

Figure 3 : Schéma du dispositif expérimental. 
N. DAN LAMSO et al. / Int. J. Biol. Chem. Sci. 9(5): 2477-2487, 2015

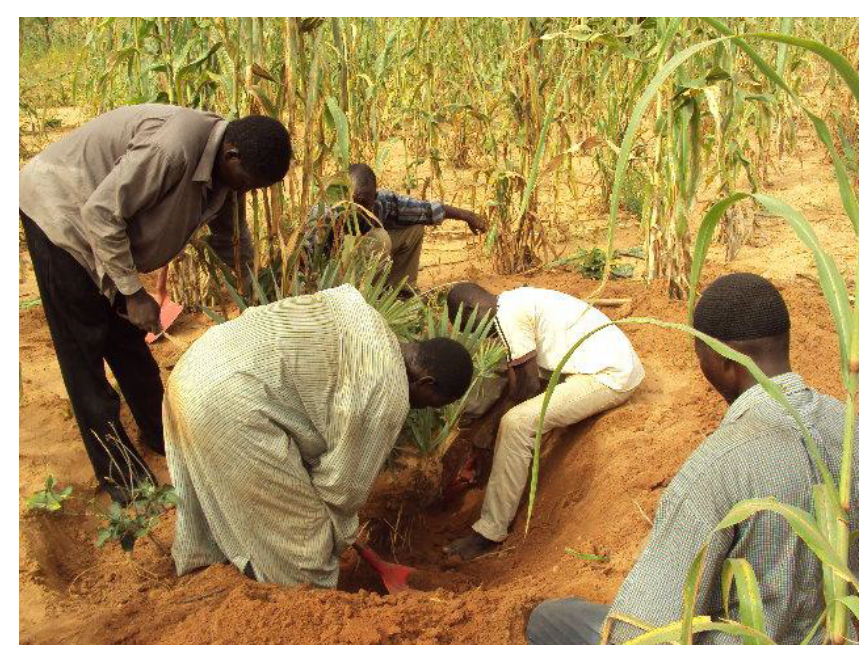

Figure 4 : Détermination de l'architecture racinaire.

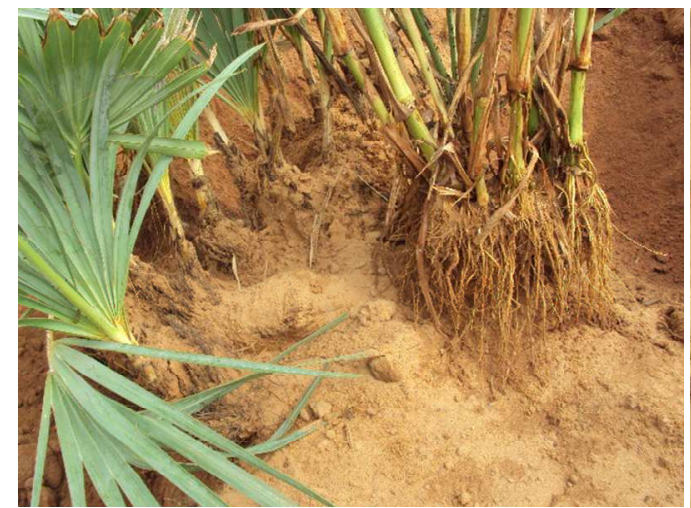

(a)

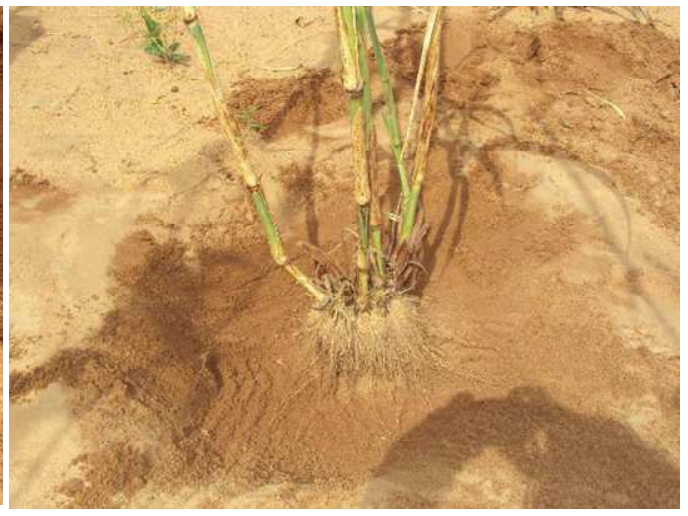

(b)

Figure 5 : Architecture racinaire du mil associé à Hyphaene thebä̈ca (a) et du mil seul (b).

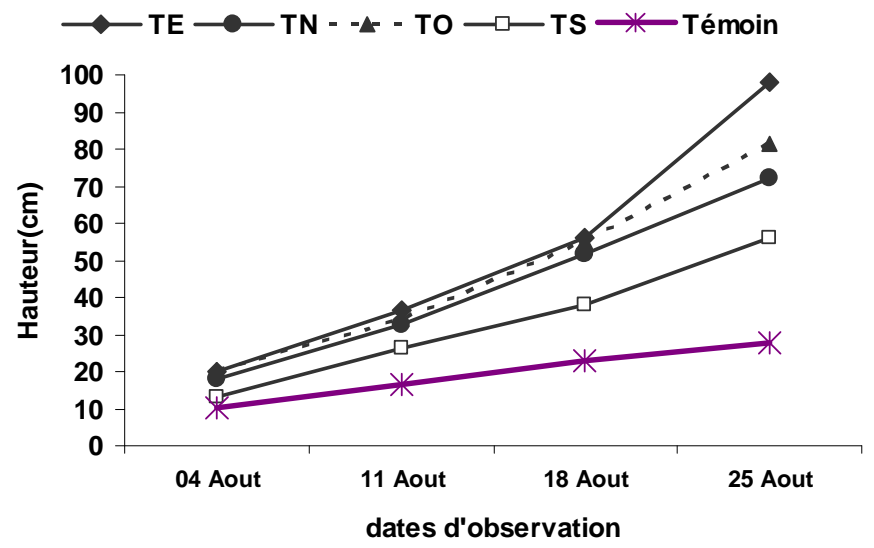

Figure 6 : Evolution de la hauteur moyenne des tiges du mil selon les directions. 


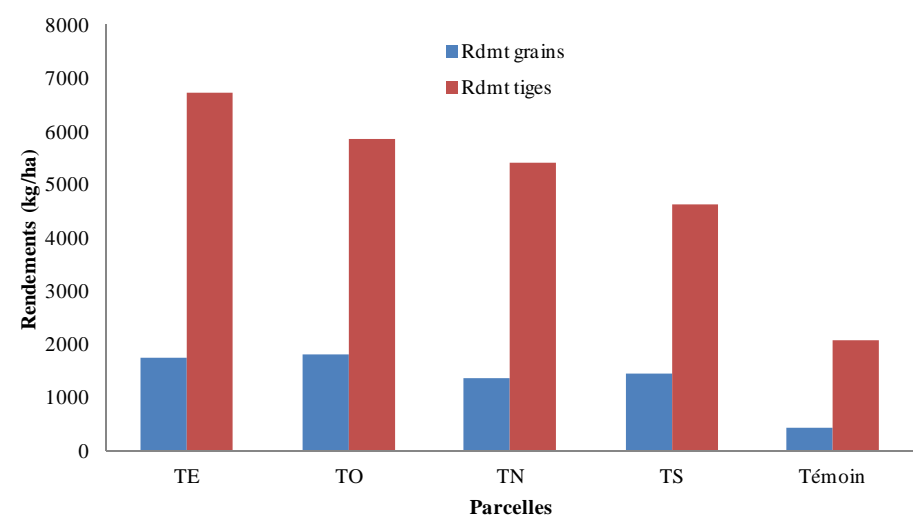

Figure 7 : Rendements en grains et tiges de mil selon les directions cardinales.

Tableau 1 : Production moyenne de talles selon les directions.

\begin{tabular}{ccc}
\hline Parcelles & Talles totales & \% talles fertiles \\
\hline TE & 96743 & 39 \\
TO & 70856 & 44 \\
TN & 96472 & 32 \\
TS & 78221 & 33 \\
Témoin & 32400 & 33 \\
\hline
\end{tabular}

\section{Conclusion}

La présente étude montre, à travers le suivi phénologique des cultures, que la présence de la touffe de Hyphaene thebaica influence favorablement le développement de la culture du mil. En effet, les observations de terrain ont permis de constater que le mil cultivé autour de la touffe de Hyphaene thebaica présente un enracinement très dense, assez profond $(40-60 \mathrm{~cm})$ et bien développé alors que le mil hors Hyphaene thebaica, présente des racines fines, superficielles (20 à $40 \mathrm{~cm}$ ), moins denses et émisses latéralement. En plus, les résultats obtenus ont montré que la croissance et les rendements de la culture du mil sont nettement plus importants dans les parcelles couvertes de Hyphaene thebaica que dans la parcelle témoin. Ainsi, la production en grains autour de Hyphaene thebaica est 3 à 4 fois (1300 à $1820 \mathrm{~kg} / \mathrm{ha}$ ) plus élevée que celle du témoin (440 kg / ha). Il en est de même pour la production en paille avec respectivement 4630 à $6740 \mathrm{~kg} / \mathrm{ha}$ et 2000 $\mathrm{kg} / \mathrm{ha}$ pour les parcelles sous influence de Hyphaene thebaica et la parcelle témoin. Il apparaît clairement que les touffes de Hyphaene thebaica favorisent la croissance et le développement du mil dans le terroir d'El Guéza, et améliore conséquemment sa production.

\section{REFERENCES}

Ambouta JMK, Issaka A, Issa S. 1998. Gestion de la fertilité des sols et évolution des sols de Gakudi (Maradi, Niger). Cahiers Agricultures, 7 : 395 - 400 p.

Bationo BA, Kalinganiré A, Bayala J. 2012. Potentialités des ligneux dans la pratique de l'agriculture de conservation dans les zones arides et semi arides de l'Afrique de l'Ouest : Aperçu de quelques systèmes candidats. ICRAF Technical Manual no. 
17 Nairobi : World Agroforestry Centre, $32 \mathrm{P}$.

Boyer J. 1978. Le Calcium et le Magnésium dans les Sols des Régions Tropicales Humides et Sub-Humides. Initiations Documentations techniques. ORSTOM : Paris ; 173.

Dan Lamso N. 2002. Valorisation des eaux de ruissellement par des techniques traditionnelles d'aménagement des sols : expériences en zones arides et semi arides méditerranéennes et sahéliennes et exemple d'efficacité au Niger. Thèse de Doctorat. Faculté des Sciences de Niamey et de Tunis. Université Abdou Moumouni de Niamey - Université de Tunis II.

Dan Lamso N, Guéro Y, Tankari Dan-Badjo A, Rabah L, André BB, Patrice D, Tidjani AD, Ado Maman N, Ambouta JM . 2015. Variations texturales et chimiques autour des touffes de Hyphaene thebaica (MART) des sols dans la région de Maradi (Niger). Algerian Journal of Arid Environment, 5(1): 40-55.

De Grandi 1996. L'évolution des systèmes de production agropastorale par rapport au développement rural durable dans les pays d'Afrique soudano-sahélienne. Collection FAO : Gestion des exploitations agricoles. 162 pages.

Dramé AY, Berti F. 2008. Les enjeux socioéconomiques autour de l'agroforesterie villageoise à Aguié (Niger). Tropicultura, 26(3): 141-149.

Harouna M. 2005. Étude de la filière des produits du palmier doum en perspective de mise en place d'un marché rural au niveau de la grappe de El Guéza (AGUIE). Mémoire de DESS CRESA, Projet PAIIP/FIDA, Niamey, 87.

Jahiel. M. 1993. Le palmier doum (Hyphaene thebaica Mart.1. IR) flamboyant. Bulletin de Liaison des Membres du Réseau Arbres Tropicaux. 28: 4-10.
Kadadé A. 1999. Système de production et gestion de la fertilité des sols dans la rôneraie de Gaya : cas de terroir de Bana, mémoire de fin d'étude / CRESA / Niamey, $68 \mathrm{p}$.

Mai Moussa KA. 1996. Environnement de Faidherbia albida Del; caractérisation, exploitation et perceptive d'optimisation dans les zones soudano-sahélienne de l'Afrique de l'Ouest; thèse de $3^{\mathrm{e}}$ cycle pour le doctorat université de Cocody / Cote d'Ivoire, 137p.

Maï Moussa KA, Williams JH, Odongo JCW. 1997. Diversification des cultures sous Faidherbia albida en milieu paysan dans la zone semiaride de l'Afrique de l'ouest. In Soil fertility management in west African land use systems. Proceedings of the regional workshop, Renard G, Neef A, Beckert $\mathrm{K}$, von Oppen M (eds).. Margraf verlag. Niamey, Niger 4-9 March 1997. p. 299-303.

Marchal M. 1983. Les paysages agraires de Haute-Volta: Analyse structurale par la méthode graphique. Atlas des structures agraires au Sud du Sahara 18. Collection publiée sous le patronage de la maison des sciences de l'Homme. ORSTOM. 99 pages + annexes.

Maydell HJ. 1981. Arbres et arbustes du Sahel : leurs caractérisations et leurs utilisations, 312-315.

Moussa H. 1997. Germination du palmier doum (Hyphaene thebaica Mart) et analyse de son interaction avec le mil (Pennisetum glaucum) en zone semi-aride au Niger. Thèse de l'Université de Laval, Québec, $181 \mathrm{p}$.

Ounténi IA. 1993. Les "Parcs" agro forestiers au Niger: état des connaissances et perspectives de recherches. Rapport de consultation. 75P.

Peltier R, Duhem CS, Ichaou A. 2008. «Valoriser les produits du palmier doum pour gérer durablement le système 
N. DAN LAMSO et al. / Int. J. Biol. Chem. Sci. 9(5): 2477-2487, 2015

agroforestier d'une vallée sahélienne du Niger et éviter sa désertification », VertigO - la revue électronique en sciences de l'environnement, 8(1), URL : http:// vertigo.revues.org/1452; DOI : 10.4000/vertigo.1452.

Roose E. 1974. Introduction à la gestion conservatoire de l'eau, de la biomasse et de la fertilité des sols (GCES). Bull. Pédol. FAO (Rome), 70: 420 p.
Tidjani Adamou D. 2008. Erosion éolienne dans le Damagaram Est (Sud-Est du Niger) : paramétrisation, quantification et moyens de Lutte, Doctorat en Sciences agronomiques et ingénierie biologique, Université Catholique de Louvain, Belgique.

Wezel A. 2000. Scattered shrubs in pearl millet fields in semiarid Niger: Effect on millet production. Agroforestery Systems, 48: 219-228. 